\title{
Succinate dehydrogenase deficient gastrointestinal stromal tumor in a three month old boy with a fatal clinical course: a case report and review of literature
}

Bei-Bei Lv ${ }^{1,2}$, Jia-Mei Li ${ }^{1,2}$, Zhi-Gang Yao ${ }^{1,2}$, Xian-Kui Cheng ${ }^{1,2}$, Fu-Xin Ren³, Wen-Jing Su, ${ }^{1,2}$, Ye-Jun Qin ${ }^{1,2}$, Zhou Wang ${ }^{1,2^{*}}$ and Zhi-xin Cao ${ }^{1,2^{*}}$

\begin{abstract}
Background: Succinate dehydrogenase deficient gastrointestinal stromal tumors (SDH-deficient GISTs), which lack KIT or PDGFRA mutations demonstrate unique clinical and pathological features, and they respond poorly to standard targeted therapy. We herein present a novel case of SDH-deficient GIST in a three-month-old infant's colon mesentery, and he is the youngest patientto date.

Case presentation: The infantpresented with complaints of blood in the stool. CT showed a $6.3 \times 4.6 \mathrm{~cm}$ mass in the left lower retroperitoneal. Complete resection of tumor and segmental bowel resection was performed without regional lymphadenectomy. Histologically, tumor cells were distinctive in their multinodular colon wall involvement with interspersed tracts of colon wall smooth muscle. The tumor was composed mainly of epithelioid cells. Immunohistochemically, the tumor cells were positive for Vim, CD117, PDGFR, while negative for SDHB. Mutational analysis showed a synonymous mutation for SDHB and wild-type for KIT and PDGFRA. Two months after surgery, metastases were found and Imatinib was administered. Unfortunately, the disease continued to progress, and the infant died 5 months after surgery.

Conclusions: SDH-deficient GISTs comprise a subgroup of a relatively rare tumor type and show a number of clinically and biologically unique features, especially for infants. It is of great importance to developing new therapeutic targets and novel specific drugs.
\end{abstract}

Keywords: SDHB, GIST, Infant, Genetic characteristics, Case report

\footnotetext{
*Correspondence: boatw@163.com; caozhixin12@126.com
}

1 Department of Pathology, Shandong provincial hospital affiliated to

Shandong First Medical University, No. 324 Jing Wu Road, Jinan 250021,

Shandong Province, China

Full list of author information is available at the end of the article

(c) The Author(s). 2021 Open Access This article is licensed under a Creative Commons Attribution 4.0 International License, which permits use, sharing, adaptation, distribution and reproduction in any medium or format, as long as you give appropriate credit to the original author(s) and the source, provide a link to the Creative Commons licence, and indicate if changes were made. The images or other third party material in this article are included in the article's Creative Commons licence, unless indicated otherwise in a credit line to the material. If material is not included in the article's Creative Commons licence and your intended use is not permitted by statutory regulation or exceeds the permitted use, you will need to obtain permission directly from the copyright holder. To view a copy of this licence, visit http://creativecommons.org/licenses/by/4.0/ The Creative Commons Public Domain Dedication waiver (http://creativecommons.org/publicdomain/zero/1.0/) applies to the data made available in this article, unless otherwise stated in a credit line to the data. 


\section{Background}

Gastrointestinal stromal tumors (GISTs) are the most common mesenchymal tumors of the gastrointestinal tract which have been recognized as genetically and biologically heterogeneous tumors. Most GISTs harbor activating mutations of KIT or platelet-derived growth factor receptor alpha (PDGFRA) [1]. Approximately 15\% of GISTs in adults and more than $90 \%$ of pediatric GISTs lack these tyrosine kinase mutations and they are generally classified as "wildtype" (WT) GISTs [1, 2]. Among them, succinate dehydrogenase (SDH)-deficient GISTs, which are associated with SDH deficiency by immunohistochemistry (IHC), are the largest group [3]. SDH-deficient GISTs occur exclusively in the stomach and are characterized by a distinctive multinodular/ plexiform architecture and epithelioid or mixed epithelioid and spindle cell morphology $[4,5]$. A small subset of patients with SDH-deficient GIST accompanies with Carney-Stratakis syndrome or Carney triad [6]. We herein present a novel case of SDH-deficient GIST in a three-month-old infant's colon mesentery, exhibiting all the clinical, morphological, immunohistochemical, and genetic characteristics of this rare tumor, followed by a brief discussion on this rare entity. To our knowledge, this is the youngest case reported to date.

\section{Case presentation}

A three-month-old baby boy presented with complaints of blood in the stool with a duration of more than half a month. On clinical examination, B-ultrasound results suggested that solid hypoechoic mass was detected in the left lower abdomen, the size was about $6.9 \times 5.0 \times$ $3.9 \mathrm{~cm}$. Enhanced Computed tomography (CT) showed a $6.3 \times 4.6 \mathrm{~cm}$ mass in the left lower retroperitoneal (Fig. 1a). The border is clear, showing uneven reinforcement. No other physical abnormalities were found. Blood routine examination revealed that hemoglobin was $63 \mathrm{~g} / \mathrm{L}$ and the red blood cell count was $2.15 \times 10^{12} / \mathrm{L}$. The baby's parents and elder sister are in good health and no related neoplastic lesions were found.

Complete resection of the tumor and segmental bowel resection was performed without regional lymphadenectomy in the pediatric surgery department. The tumor located in the left mesentery with invasion into the colon and sigmoid junction. Adjuvant anti-cancer treatment was not undertaken after the operation on account of bad general condition.

Microscopically, under low magnification, tumors were distinctive in their multinodular colon wall involvement with interspersed tracts of colon wall smooth muscle (Fig. 2a), and this is often referred to a "plexiform" pattern as reported before. Under high magnification, the tumor cells have mainly epithelioid cytology with variably eosinophilic cytoplasm. The nucleus was round or oval, and the nucleolus was obvious. Mitotic images were easy to be seen, mitotic count $/ 5 \mathrm{~mm}$ (squared) is more than 50. (Fig. 2b). Lymphovascular and nerve invasion were not found under the microscope.

The panel of immune-histochemical stains included CD117, Dog1, CD34, SDHB, S100, SMA, Desmin, Vim, and Ki67. Among them, Vim, CD117, and PDGFR were diffusely and strongly positive (Fig. 2c, d), which supports the diagnosis of gastrointestinal stromal tumors. Besides, the tumor cells lack SDHB expression, but normal intestinal mucosa and vascular elements were positive which verify adequate immunohistochemical detection (Fig. 2e). CD34, Dog1, S100, SMA, Desmin, were negative. The Ki67 labeling index (MIB-1 index) reached $30 \%$ in the area of greatest concentration (Fig. 2f).

Mutational analysis showed a wild-type for KIT and PDGFRA at the five exons examined (KIT exons 9,11,13, 17 and PDGFRA exon 18). In addition, all other targets (Her2、EGFR、RET、ROS1、PI3KCA、ALK、K-

RAS、NRAS and MET) showed no mutation. However, CCND2 amplification and amino acid missense mutation at position 932 of exon 19 of the PTCH1 gene were
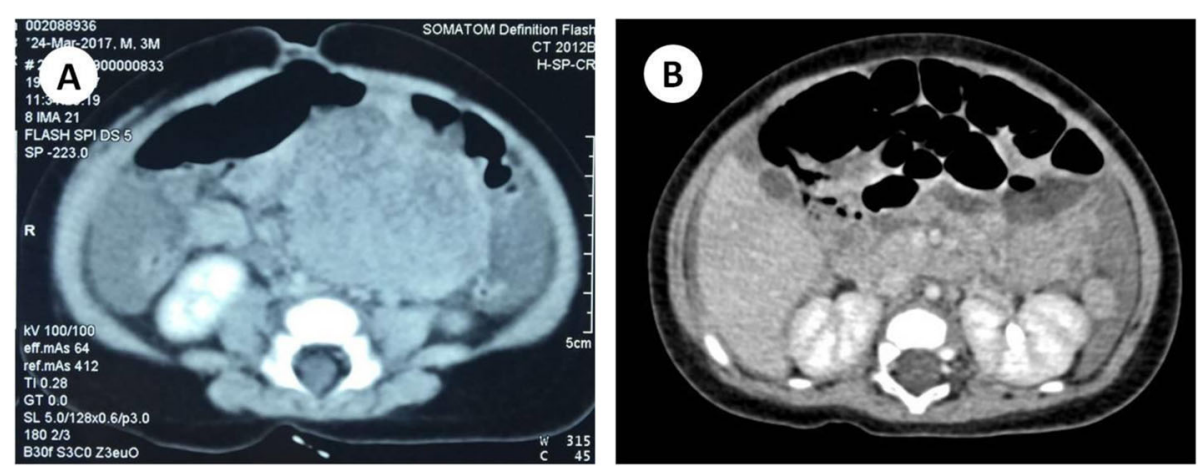

Fig. 1 Radiologic findings of the horizontal views. a: Enhanced CT showing a $6.3 \times 4.6 \mathrm{~cm}$ mass in the left lower retroperitoneal with a clear boarder. b: Enhanced CT findings suggested that multiple metastases were seen in retroperitoneal 


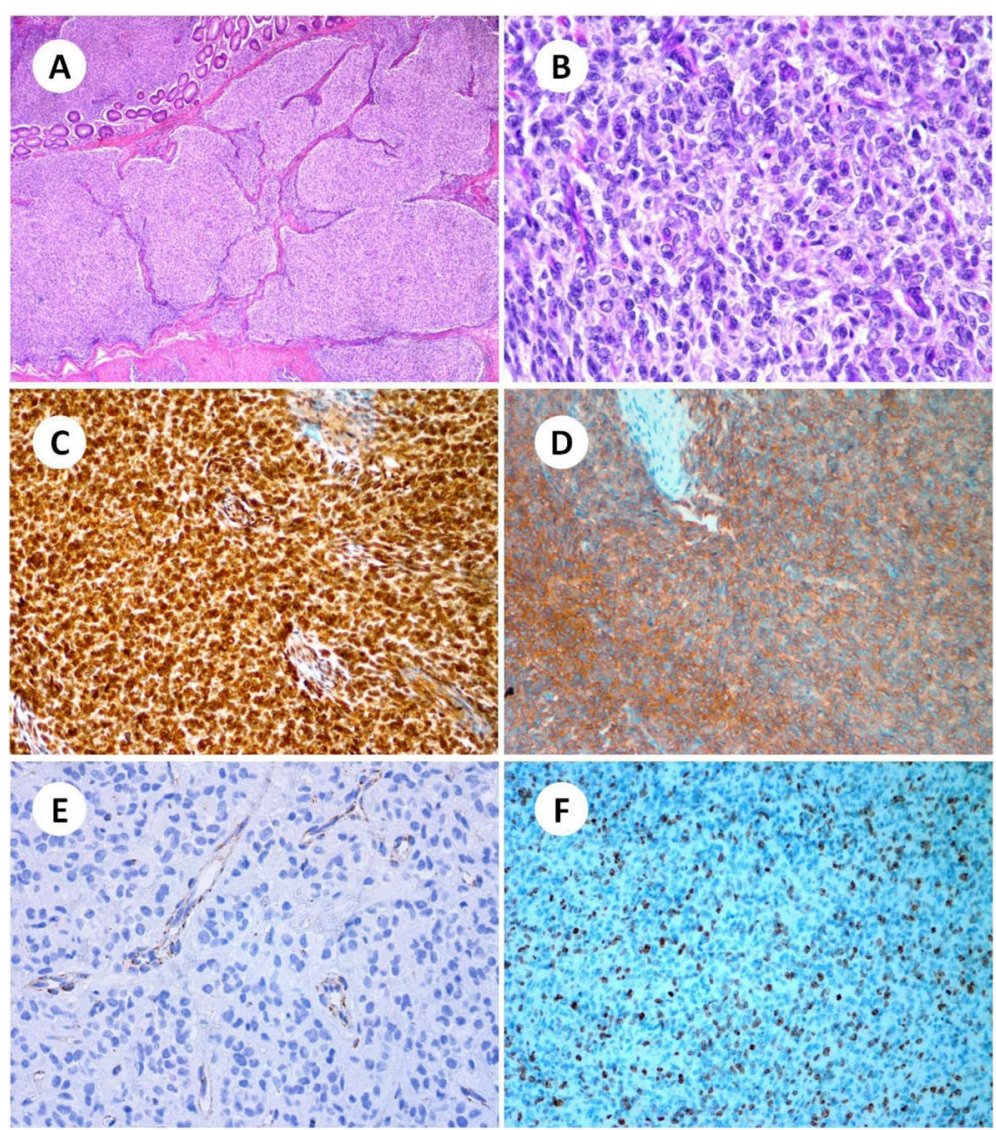

Fig. 2 The microscopic features and immunohistochemical stains of the lesion. a: Tumors are distinctive in their multinodular colon wall involvement with interspersed tracts of colon wall smooth muscle $\left(H \& E_{1} \times 100\right)$. $\mathbf{b}$ : The tumor cells have a mainly epithelioid cytology with variably eosinophilic cytoplasm. Mitotic images were easy to be seen $\left(H \& E_{1} \times 400\right)$. c: PDGFR was diffusely and strongly positive $(\times 200)$. d: The tumor cells were positive for CD117 (× 200). e: The tumor cells lack SDHB expression, but vascular elements were positive $(\times 400)$. f: The Ki-67 labelling index (MIB-1 index) reached $30 \%$ in the area of greatest concentration $(\times 200)$

detected, which may have a significant impact on gene function. At last, we performed SDHB gene sequencing in Jinan Boshang Biotechnology Co. Ltd. The sequencing of SDHB in tumor showed synonymous mutation at position 169 of exon $1(\mathrm{C}-\mathrm{A}$ ) (Fig. 3), which may be related to the occurrence and development of this tumor.

Based on these findings, the pathological diagnosis of SDH-deficient GIST was established.

Two months later, the infantwas brought to our hospital again presented with complaints of cough for 2 weeks and diarrhea for 2 days. The results of abdominal color Doppler ultrasound showed that there was much effusion in the abdominal cavity. Abdominal enhanced CT findings suggested that multiple metastases were seen in the peritoneum, mesentery, retroperitoneal, left groin, and right lower abdominal wall (Fig. 1b). Pulmonary $\mathrm{CT}$ suggested double lung inflammatory lesions. Anti-infective treatment was carried out in PICU to correct symptomatic and supportive treatment such as anemia. When the condition improved, the infant left our hospital. According to the advice of Zhongshan First Affiliated Hospital, Imatinib (100 mg, once daily) was administered. Because of severe diarrhea, the medicine was withdrawal after 3 weeks. Unfortunately, the disease continued to progress, and the infant died 5 months after surgery.

\section{Discussion}

SDH-deficient GISTs represent the largest proportion of WT GISTs that lack KIT or PDGFRA mutations. The true frequency of SDH-deficient GISTs was reported to be approximately 7.4 to $7.7 \%$ [6-8]. This group encompasses most pediatric GISTs and two previously described syndromes: Carney-Stratakis syndrome and Carney triad.

Succinate dehydrogenase (SDH) is an enzyme complex composed of four protein subunits (SDHA, SDHB, SDHC, and SDHD). This complex acts at the interphase of the tricarboxylic acid cycle and electron transport chain. The SDH-complex participates in the Krebs cycle 


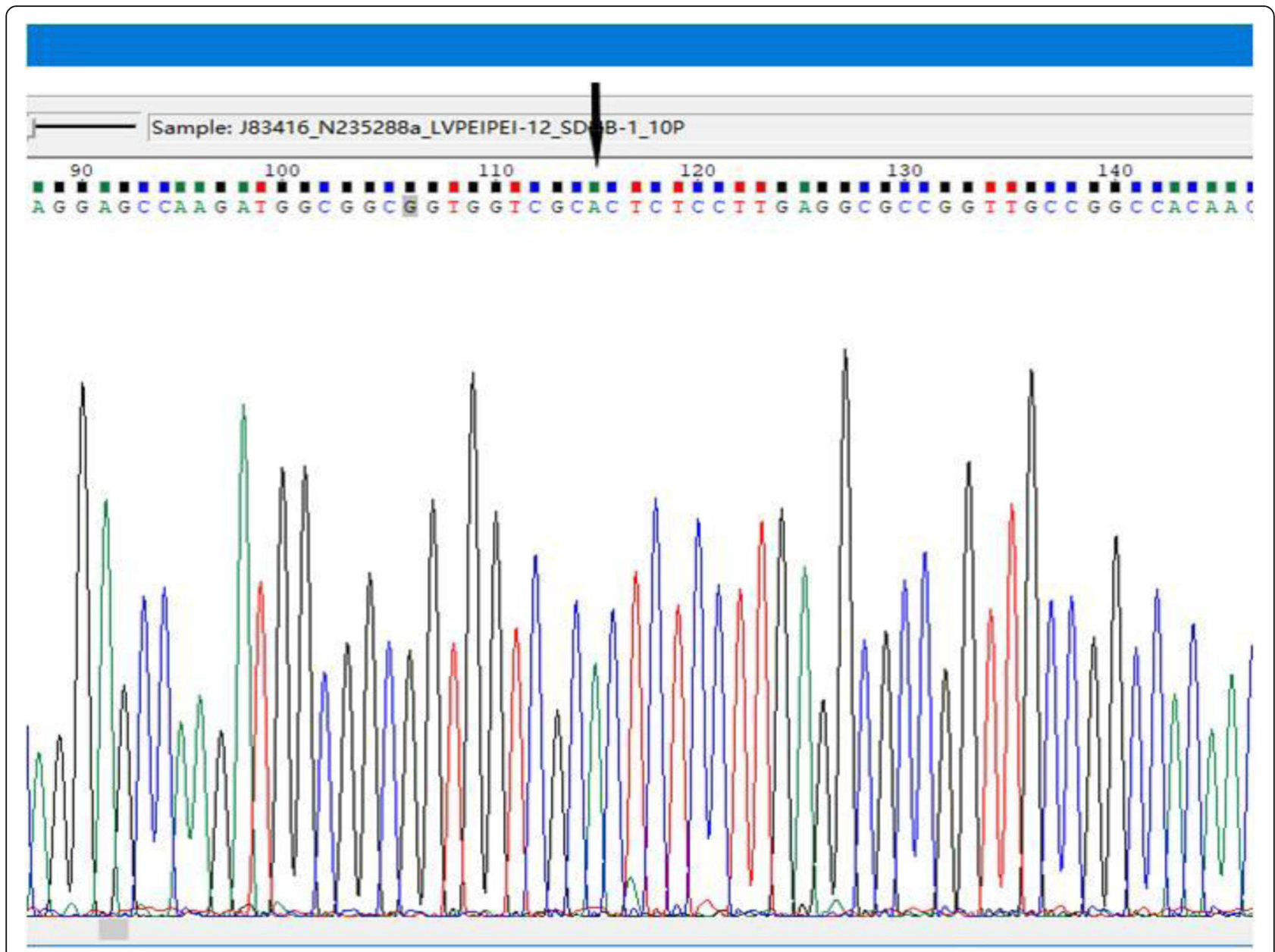

Fig. 3 Sequencing results shows mutation at position 169 of exon 1(C-A) ( $\downarrow$ shows mutated loci)

with subunit A (SDHA) being the catalytic unit responsible for the conversion of succinate to fumarate. Subunit $\mathrm{B}(\mathrm{SDHB})$ is an iron-sulfur protein that participates in the electron transport chain for the oxidation of ubiquinone to ubiquinol. Subunits C and D (SDHC and SDHD) are membrane-anchoring subunits. Remarkably, immunohistochemistry for SDHB becomes negative whenever there is bi-allelic inactivation of any component of SDH, which is very rare in the absence of syndromic disease [9]. Loss of SDHB, as tested by immunohistochemistry, is the most practical way to identify SDH-deficient tumors [3, 7]. Loss of function of the succinate dehydrogenase complex characterizes a rare group of human tumors including some gastrointestinal stromal tumors, paragangliomas, renal carcinomas, and pituitary adenomas. They can all be characterized as SDH-deficient tumors $[9,10]$.

SDH-deficient GISTs demonstrate unique clinical and pathological features, including an exclusively gastric location, absence of KIT or PDGFRA mutations, typically showed plexiform muscularis propria involvement, and epithelioid hypercellular morphology. Based on current experience, SDH-deficient GISTs occur mainly in the stomach and were more likely to occur in younger, female patients. In this report, we showed a colon mesentery tumor case in a three-month-old infant, which was the youngest patient also in an unusual location reported to date $[3,7,8,11]$. Malignant tumors in infants and young children may represent a different subgroup because of their unique clinical features and biological behavior. The cause of the tumor is unclear. Whether it is associated with embryonic development needs more cases to study.

SDH-deficient GISTs are characterized by a distinctive multinodular/ plexiform architecture and epithelioid or mixed epithelioid and spindle cell morphology [4]. In our case, the "plexiform" pattern is apparent, and the tumor cells have mainly epithelioid cytology, which is consistent with the reports before and support for the diagnosis. Not surprisingly, IHC examinations for SDHdeficient GISTs showed positivity for CD117, CD34, DOG-1 and PDGFR, and negativity for SMA and S-100. 
Loss of SDHB expression is a consistent feature of SDHdeficient GISTs. The most important molecular change of SDH-deficient GISTs is SDH mutations followed by SDHC promoter hypermethylation [3, 12]. SDH mutations are often germline and most commonly A (about $30 \%$ ), and $\mathrm{B}, \mathrm{C}$, or $\mathrm{D}$ (together $20 \%$ ) $[3,6,13]$. It is unknown how mutations in the various $\mathrm{SDH}$ subunits may differentially regulate tumor biology. Patients with alterations of the SDHC gene may be less likely to develop distant metastases [6]. Besides, another feature of SDHdeficient GISTs is overexpression of insulin-like growth factor 1 receptor (IGF1R) gene, possibly by gene amplification. Chou A et al [14] used immunohistochemistry suggested that IGF1R is overexpressed in $100 \%$ of SDHdeficient GISTs but never in non-SDH deficient GISTs. In our case, the sequencing of SDHB showed synonymous mutation at position 169 of exon $1(\mathrm{C}-\mathrm{A})$ and the mutation site agrees with the results of the previous study [15]. Although amino acid has not changed, synonymous mutation frequently acts as driver mutations in human cancers. Fran Supek et al. present robust statistical evidence in an analysis of $>3000$ cancer exomes and $>300$ cancer genomes that synonymous mutations in exons may act through diverse molecular mechanisms, and are often associated with changes in splicing [16]. Marc Bennedbæk et al. identified SDHB, SDHC, SDHD germline variants in Danish pheochromocytoma/ paraganglioma patients and founded that all three SDHB missense variants were predicted as pathogenic. In silico splicing analysis indicates that this change could affect the splicing of exon 5 [17]. Therefore, we speculate synonymous mutation of SDHB in our study may be pathogenic through the same mechanism. Besides, the tumor has an SDHA or SDHC alteration is also possible.

In addition, CCND2 amplification and amino acid missense mutation at position 932 of exon 19 of the PTCH1 gene was found. CCND2 belongs to the highly conserved cyclin family, forms a complex with CDK4 or $\mathrm{CDK} 6$, and functions as a regulatory subunit of the complex, whose activity is required for cell cycle G1/S transition. CCND2 amplification has been previously reported in GIST [18]. PTCH1 encodes a member of the patched family of proteins and a component of the hedgehog signaling pathway, which is important in embryonic development and tumorigenesis [19]. Whether the changes in these two genes are related to the tumor needs more cases for further study.

SDH-deficient GISTs do not seem to have a marked tendency for familial occurrence, as compared with SDH mutation syndrome associated paragangliomas that show familial occurrence. Due to the rarity of SDH-deficient GISTs, treatment experience is limited, especially for pediatric patients. Complete surgical removal of the primary tumor and locoregional (omental or nodal) metastases should be performed whenever possible. There is no uniform about the adjuvant treatment of patients with SDH-deficient GISTs to date. Traditional cytotoxic chemotherapy is generally ineffective for $\mathrm{SDH}$ deficient GISTs, as it is for KIT/PDGFRA mutant GISTs. SDH-deficient GISTs respond poorly to standard targeted therapy such as tyrosine kinase inhibitor drugs, neither the first line inhibitor imatinib mesylate nor a second line multikinase inhibitor sunitinib malate, although the stable disease has been observed in some cases $[6,8,20]$. Newer tyrosine kinase inhibitor drugs are potentially usable in SDH-deficient GISTs include regorafenib, nilotinib, and sorafenib [21]. Better molecular and clinical characterization could improve management.

Conventional risk stratification fails to predict the progression of SDH-deficient GISTs [6]. SDH deficient GISTs run a relatively indolent course despite their frequent lymph node or distant metastasis. Follow-up data shows some patients survived for $10 \sim 17$ years after peritoneal metastases [7]. However, our case only survived for 5 months after surgery, which may be related to the unique nature of infant and young child malignancies. This case reminds us that such tumors should be vigilant and it is necessary to collect more cases to study the appropriate treatment.

\section{Conclusions}

Consequently, SDH-deficient GISTs comprise a subgroup of a relatively rare tumor type and show some clinically and biologically unique features. It is of great importance to developing new therapeutic targets and novel specific drugs. The priority for further research in this molecular subtype is more extensive sequencing with methods such as whole-exome sequencing, RNA sequencing, and whole-genome sequencing to discover novel genomic events affecting kinases that could suggest therapeutic vulnerabilities. We reported the youngest case of SDH-deficient GIST arising in colon mesentery, and reviewed the relevant literature to make a deeper understanding of the disease, and provide useful parameters for further gene therapy.

\section{Abbreviations \\ GIST: Gastrointestinal stromal tumors; SDH: Succinate dehydrogenase; $\mathrm{CT}$ : Computed tomography; IHC: immunohistochemistry; WT: wildtype}

Acknowledgements

Not applicable.

\section{Authors' contributions}

LV BB and Wang Z designed the study; Li JM and Yao ZG acquired clinical data; Su WJ and Cheng XK performed the pathological examination; Ren FX performed the image examination; Lv BB wrote the manuscript; Qin YJ, Wang $Z$ and Cao ZX revised the manuscript. All authors issued final approval for the version to be submitted. 


\section{Funding}

Supported by the Shandong Province Key R\&D Program 2019GSF108268.

\section{Availability of data and materials}

All data generated or analyzed during this case are included within the article.

\section{Ethics approval and consent to participate}

The need for ethics approval and consent was waived, since a consent for publication was obtained from the patient's parents.

\section{Consent for publication}

Written informed consent for publication of the clinical details and/or clinical images was obtained from the parents of the patient. A copy of the consent form is available for review by the Editor of this journal.

\section{Competing interests}

The authors declare that they have no competing interests.

\section{Author details}

'Department of Pathology, Shandong provincial hospital affiliated to Shandong First Medical University, No. 324 Jing Wu Road, Jinan 250021, Shandong Province, China. ${ }^{2}$ Department of Pathology, Shandong Provincial Hospital affiliated to Shandong University, Jinan 250021, Shandong Province, China. ${ }^{3}$ Shandong Medical Imaging Research Institute, Shandong University, Jinan 250021, Shandong Province, China.

Received: 1 September 2020 Accepted: 15 February 2021

Published online: 21 February 2021

\section{References}

1. Doyle LA, Hornick JL. Gastrointestinal stromal tumours: from KIT to succinate dehydrogenase. Histopathology. 2014;64(1):53-67.

2. Wang YM, Gu ML, Ji F. Succinate dehydrogenase-deficient gastrointestinal stromal tumors. World J Gastroenterol. 2015;21(8):2303-14.

3. Boikos SA, Pappo AS, Killian JK, et al. Molecular subtypes of KIT/PDGFRA wild-type gastrointestinal stromal tumors: a report from the National Institutes of Health gastrointestinal stromal tumor clinic. JAMA Oncol. 2016; 2(7):922-8.

4. Doyle LA, Nelson D, Heinrich MC, et al. Loss of succinate dehydrogenase subunit $B(S D H B)$ expression is limited to a distinctive subset of gastric wildtype gastrointestinal stromal tumours: a comprehensive genotypephenotype correlation study. Histopathology. 2012;61(5):801-9.

5. Ibrahim A, Chopra S. Succinate dehydrogenase-deficient gastrointestinal stromal tumors. Arch Pathol Lab Med. 2020;144(5):655-60.

6. Mason EF, Hornick JL. Conventional risk stratification fails to predict progression of succinate dehydrogenase-deficient gastrointestinal stromal tumors: a Clinicopathologic study of 76 cases. Am J Surg Pathol. 2016; 40(12):1616-21.

7. Miettinen M, Wang ZF, Sarlomo-Rikala M, et al. Succinate dehydrogenasedeficient GISTs: a clinicopathologic, immunohistochemical, and molecular genetic study of 66 gastric GISTs with predilection to young age. Am J Surg Pathol. 2011;35(11):1712-21.

8. Liu W, Zeng X, Wu X, et al. Clinicopathologic study of succinatedehydrogenase-deficient gastrointestinal stromal tumors: a singleinstitutional experience in China [J]. Medicine (Baltimore). 2017;96(32):e7668.

9. Gill AJ. Succinate dehydrogenase (SDH)-deficient neoplasia. Histopathology. 2018;72(1):106-16.

10. Miettinen M. Succinate dehydrogenase-deficient tumors--a novel mechanism of tumor formation. Duodecim. 2015;131(22):2149-56.

11. Miettinen M, Felisiak-Golabek A, Wang Z, et al. GIST manifesting as a retroperitoneal tumor: Clinicopathologic Immunohistochemical, and molecular genetic study of 112 cases. Am J Surg Pathol. 2017;41(5):577-85

12. Urbini $M$, Astolfi $A$, Indio $\mathrm{V}$, et al. SDHC methylation in gastrointestinal stromal tumors (GIST): a case report [J]. BMC Med Genet. 2015;16:87.

13. Dwight T, Benn DE, Clarkson A, et al. Loss of SDHA expression identifies SDHA mutations in succinate dehydrogenase-deficient gastrointestinal stromal tumors. Am J Surg Pathol. 2013;37(2):226-33.

14. Chou A, Chen J, Clarkson A, et al. Succinate dehydrogenase-deficient GISTs are characterized by IGF1R overexpression. Mod Pathol. 2012;25(9):1307-13.
15. Cheng $Y$, Zhang Z, Zhu H, et al. Clinicopathologic features of succinate dehydrogenase-deficient gastrointestinal stromal tumor. Zhonghua Bing Li Xue Za Zhi. 2016;45(3):153-8.

16. Supek F, Minana B, Valcarcel J, et al. Synonymous mutations frequently act as driver mutations in human cancers. Cell. 2014;156(6):1324-35.

17. Bennedbæk M, Rossing M, Rasmussen ÅK, et al. Identification of eight novel SDHB, SDHC, SDHD germline variants in Danish pheochromocytoma/ paraganglioma patients. Hered Cancer Clin Pract. 2016;14:13.

18. Saponara M, Urbini M, Astolfi A, et al. Molecular characterization of metastatic exon 11 mutant gastrointestinal stromal tumors (GIST) beyond KIT/PDGFRa genotype evaluated by next generation sequencing (NGS). Oncotarget. 2015;6(39):42243-57.

19. Regan JL, Schumacher D, Staudte S, et al. Non-canonical hedgehog signaling is a positive regulator of the WNT pathway and is required for the survival of colon cancer stem cells. Cell Rep. 2017;21(10):2813-28.

20. Pantaleo MA, Lolli C, Nannini M, et al. Good survival outcome of metastatic SDH-deficient gastrointestinal stromal tumors harboring SDHA mutations. Genet Med. 2015;17(5):391-5.

21. Miettinen M, Lasota J. Succinate dehydrogenase deficient gastrointestinal stromal tumors (GISTs) - a review. Int J Biochem Cell Biol. 2014:53:514-9.

\section{Publisher's Note}

Springer Nature remains neutral with regard to jurisdictional claims in published maps and institutional affiliations.
Ready to submit your research? Choose BMC and benefit from:

- fast, convenient online submission

- thorough peer review by experienced researchers in your field

- rapid publication on acceptance

- support for research data, including large and complex data types

- gold Open Access which fosters wider collaboration and increased citations

- maximum visibility for your research: over $100 \mathrm{M}$ website views per year

At BMC, research is always in progress.

Learn more biomedcentral.com/submissions 